\title{
Comparing the selective landscape of TLR7 and TLR8 across primates reveals unique sites under positive selection in Alouatta.
}

Nicole S. Torosin ${ }^{1}$, Hernan Argibay ${ }^{3}$, Timothy H. Webster ${ }^{1}$, Patrice Showers Corneli $^{2}$, Leslie A. Knapp ${ }^{1}$

${ }^{1}$ Department of Anthropology, University of Utah, 260 S. Central Campus Dr., Salt Lake City, UT 84112

2 Department of Biology, University of Utah, 257 S. 1400 E., Salt Lake City, UT 84112

${ }^{3}$ Instituto de Ecología, Genética y Evolución de Buenos Aires (IEGEBA-CONICET), Intendente Güiraldes 2160 - Ciudad Universitaria (C1428EGA) Ciudad Autónoma de Buenos Aires, Argentina 


\begin{abstract}
Among primates, susceptibility to yellow fever (YFV), a single-stranded (ss) RNA virus, ranges from complete resistance to high susceptibility. Howler monkeys (genus Alouatta) are the most susceptible to YFV. In order to identify Alouatta-specific genetic factors that may be responsible for their susceptibility, we collected skin samples from howler monkey museum specimens of the species A. caraya and A. guariba clamitans. We compared the rate of nonsynonymous to synonymous $(d N / d S)$ changes of Toll-like receptor (TLR) 7 and TLR8, the two genes responsible for detecting all ssRNA viruses, across the Primate order. Overall, we found that the TLR7 gene is under stronger purifying selection in howler monkeys compared to other New World and Old World primates, but TLR8 is under the same selective pressure. When we evaluated $d N / d S$ at each codon, we found six codons under positive selection in Alouatta TLR8 and two codons under positive selection in TLR7. The changes in TLR7 are unique to A. guariba clamitans and are found in functionally important regions likely to affect detection of ssRNA viruses by TLR7/TLR8, as well as downstream signaling. These amino acid differences in A. guariba clamitans may play a role in YFV susceptibility. These results have implications for identifying genetic factors affecting YFV susceptibility in primates.
\end{abstract}




\section{Introduction}

Host-pathogen interactions are major drivers of immune gene evolution (Sironi et al. 2015). Viruses, in particular, have caused close to $30 \%$ of all adaptive amino acid changes in mammals (Enard et al. 2016). Genetic comparisons

${ }_{5}$ between species can help identify substitutions differentially affecting disease susceptibility or resistance (Wlasiuk and Nachman, 2010; Areal et al., 2011. Schad and Voigt, 2016).

Among primates, susceptibility to yellow fever virus (YFV) ranges from complete resistance to high susceptibility (Table 1). Howler monkeys (genus

10 Alouatta) are the most susceptible and usually die within a week of infection (Bicca-marques and Freitas, 2010). Currently, there is no explanation for Alouatta's high level of susceptibility to YFV.

In general, to understand species-specific genetic changes in response to pathogens, researchers compare the selective landscape of immune genes (Quach

15 et al., 2013; Wlasiuk and Nachman, 2010). TLRs, such as TLR7 and TLR8, are evolutionarily-constrained innate immune genes specifically responsible for detecting single-stranded (ss) RNA viruses, such as West Nile virus, dengue virus, and YFV (Kawai and Akira, 2010, Kumar et al., 2011; Hanley et al. 2013). When these two genes detect ssRNAs, they initiate a signaling cascade

20 that prompts the immune system to destroy infected cells and activate adaptive immunity (Kumar et al., 2011).

TLR7 and TLR8 are under strong purifying selection in humans and great apes (Quach et al., 2013). Amino acid changes that do occur are likely shaped by environmental or species-specific pathogens (Wlasiuk and Nachman, 2010).

25 Polymorphisms within TLR7 and TLR8 have been implicated in progression of various human diseases such as Hepatitis $\mathrm{C}$ and Congo hemorrhagic fever (Lin et al. 2012). Therefore, TLR7 and TLR8 are good candidates to study variable susceptibility to ssRNA viral pathogens.

However, nothing is known about evolutionary patterns of endosomal TLRs

3o in Alouatta and other New World primates. To address the question of Alouatta's susceptibility to YFV, we collected skin samples from one taxidermied howler monkey of each species, A. caraya and A. guariba clamitans, and compared the selective landscape of newly sequenced TLR7 and TLR8 exons from A. caraya and A. guariba clamitans to TLR7 and TLR8 from published New World and

35 Old World primates less susceptible to YFV (Table 22. Our comparison of TLR7 and TLR8 across primate species will help identify amino acid substitutions and selection patterns that may contribute to the high susceptibility of Alouatta to YFV. 


\section{Materials and Methods}

40

Initially, we collected one Alouatta caraya liver sample from an individual that died during the 2007-2009 YFV outbreak in Misiones, Argentina as part of a YFV epizootic research project conducted by WCS-Global Health Program (Table S1). We stored the tissue at $-70^{\circ} \mathrm{C}$ in $100 \%$ ethanol. We also col45 lected $1-1.5 \mathrm{~cm}$ skin samples from one taxidermied $A$. caraya and one $A$. guariba clamitans museum specimen at the Museo Argentina de Ciencias Naturales Bernardino Rivadavia. We stored each skin sample in a sterile $1.5 \mathrm{ml}$ Eppendorf tube at ambient temperature until DNA extraction.

We transported the tissue and taxidermied skin samples to the Universidad de Misiones at Posadas, Misiones, Argentina (transport permit 005436 issued by the "Jefatura de Gabinete de Ministros Secretaría de Ambiente y Desarrollo Sustentable"). At the Universidad, we extracted DNA using a Qiagen Fast DNA tissue kit (Qiagen, GmbH, Germany). We transported extracted DNA to Buenos Aires (transport permit 001494 issued by the "Presidencia de la Nación

55 Secretaría de Ambiente y Desarrolo Sustentable"). We exported extracted DNA from Argentina using CITES export permit 042117 and CDC import permit number 2017-03-014.

To obtain DNA sequences, the University of Utah Huntsman Cancer Institute High-Throughput Genomics Center prepared and shotgun sequenced the

${ }_{60}$ A. caraya liver tissue DNA on an Illumina HiSeq 2500. The coding regions of TLR7 and TLR8 are highly conserved among primate species (Wlasiuk and Nachman, 2010), so we used BWA-MEM (Li, 2013) to align the reads from the three exons of TLR7 and two exons of TLR 8 to a human reference sequence (1000 Genomes Project Consortium et al., 2015) and an unpublished A. palliata 65 reference sequence (A. Burrell, pers. comm.) to confirm alignment. We aligned mtDNA genes ND1, ND2, and CO1 to the A. palliata reference sequence. We used SPAdes software to create an A. caraya TLR7 and TLR8 consensus sequence (Bankevich et al., 2012) (Appendix: Methods).

Using the newly generated $A$. caraya TLR7 and TLR8 reference sequences,

70 we created a custom Ampliseq library IAD149391-168 (LifeTechnologies, Austin, TX). The University of Utah Sequencing Core completed targeted sequencing of the A. caraya and A. guariba clamitans skin samples using an Ion PGM and standard library kit protocols. To process the sequencing reads, we first trimmed the low quality bases from the ends and filtered fastq reads to maq $>20$ 75 using BBDuk (http://jgi.doe.gov/data-and-tools/bb-tools/). Next we aligned trimmed fastq reads for each sample to the A. caraya TLR7 and TLR8 reference sequences using BWA-MEM (Li, 2013). We used Freebayes (Garrison and Marth, 2012) to call variants for each sample. We used vcftools to remove indels and filter all sites with GQ $<30$ (Danecek et al. 2011). We used bcftools to

so create a consensus sequence for each taxidermied skin sample (Li et al., 2009) (Appendix: Methods). GenBank accession numbers for all sequences generated can be found in Table $\mathbf{S 2}$ 
DNA damage results in deamination of cytosine and guanine resulting in and excess of tyrosine and adenine substitutions, especially at the ends of sequencing reads (Briggs et al., 2007). Museum tissue samples are old and often subject to DNA damage, therefore we measured DNA damage using mapDamage2.0 software (Jónsson et al., 2013).

\subsection{Published data and sequence alignment}

We obtained coding regions of TLR7 and TLR8 for New World and Old World primates from GenBank and dnazoo.org (Dudchenko et al., 2017) (Table S3). We aligned DNA sequences using MEGA7 (Kumar et al. | 2016) and confirmed amino acid translation of TLR7 and TLR8 using protein reference NP 057646.1 and NP 619542.1, respectively. We aligned and edited TLR7 and TLR8 amino acid translations for all species using Aliview (Larsson, 2014).

\subsection{Phylogenetic analyses}

Optimal phylogenetic models for each gene were chosen on the basis of the Decision-Theoretic (DT) method (Sullivan and Joyce, 2005) as implemented in PAUP 4.0 (Swofford, 2003) for model selection and estimating parameters (Table S4). We optimized the trees using these models in the Seaview implementation of PhyML (Guindon et al., 2010). Approximate likelihood ratio tests (aLRT) assign clade support levels across the tree (Anisimova and Gascuel, 2006).

\subsection{Maximum Likelihood Selection Analysis}

To test the hypothesis that the Alouatta lineage evolved under a different se-

lection regime from other primates, we used the the positive selection option in HyPhy to "test whether a branch (or branches) in the tree evolve under different $d N / d S$ than the rest of the tree" (HyPhy software (Kosakovsky Pond et al. 2005)). This analysis assumes a Muse Gaut codon model and is designed to test whether the ratio of non-synonymous to synonymous $(d N / d S)$ substitu-

110 tions is equal across the phylogenetic tree or is best described by multiple rates (Frost et al., 2005; Muse and Gaut, 1994). We compared selection coefficients of the following bipartitions: 1) the branch leading to newly sequenced species A. guariba clamitans and $A$. caraya only; 2) the branch leading to the whole Alouatta genus; and 3) the branch leading to all other New World primates 115 (Figure S1).

A Fixed Effect Likelihood (FEL) test (HyPhy software (Kosakovsky Pond et al., 2005)) identified specific codons under selection in the Alouatta lineage. The null model assumes that the selection pressure for each site is constant for the entire alignment. The alternative model allows the $d N / d S$ rate to vary site

120 by site along the alignment. Results indicate whether certain sites in a selected lineage have been subject to positive or purifying selection. This analysis is designed for small sample sets and minimizes false positives (Kosakovsky Pond 
and Frost, 2005). We also completed FEL analysis for all other New World primates and Old World primates to see whether sites under selection in Alouatta are unique to the lineage.

\subsection{Functional assessment}

We assessed codon sites that showed evidence of positive selection for functional purpose. The TLR7 ligand binding region is within codons 500-589 in protein reference NP 057646.1 and within codons 481-577 in protein reference NP 619542.1 for TLR8 (Wei et al., 2009). Leucine-rich repeats (LRR), non-LRR insertions, and the Toll-interleukin receptor (TIR) domain for TLR7 and TLR8 are outlined in Bell et al. (2003). We examined codon sites in LRR, non-LRR insertions, and TIR domains (Bell et al. 2003) to determine if there was evidence for positive selection within any of the binding, LRR, insertion, or TIR 135 regions.

\section{Results}

\subsection{Sequences}

We generated two A. caraya sequences and one A. guariba clamitans sequence for nuclear genes TLR7, exons 1 (221 bp), 2 (196 bp), and 3 (5635 bp); TLR8, 140 exons 1 (201 bp) and 2 (4217 bp); and mtDNA genes ND1 (1075 bp), ND2 (1145 bp), and CO1 (1742 bp). Average sequencing coverage is reported in Table S5, Table S6. We did not detect post-mortem DNA damage in the museum samples (Table S7).

\subsection{Phylogenetic trees}

145 Maximum likelihood phylogenetic trees (Figure 1, Figure 2) show Alouatta species clustering next to other New World primates. The separate clustering of Alouatta is supported by other studies using nuclear genes (Steiper and Ruvolo, 2003: Boissinot et al., 1998; Harada et al., 1995) and mtDNA (Figure [S2). Old World primates cluster as anticipated (Pozzi et al., 2014). In both nuclear

DNA trees, the A. guariba clamitans branch length is longer than the other two Alouatta species, and in TLR7, it is the longest in the entire tree (Figure 1 . Figure 2).

\subsection{Substitution rates}

We used a maximum likelihood approach to determine whether Alouatta branches

evolved under a different $d N / d S$ ratio from the rest of the primate tree. For this test, significance is reached at 0.10 because the standard 0.05 cutoff is too conservative and eliminates too many observations that deviate from the null hypothesis (Kosakovsky Pond and Frost, 2005). Our null hypothesis was that $d N / d S$ is the same across the entire phylogenetic tree (Frost et al., 2005). We 
compared the following tree partitions: branches leading to the A. caraya and A. guariba clamitans clade, the branch leading to the whole Alouatta clade, the branch leading to the other New World primates, as well as the branch leading to the Old World primates (see Figure $\mathbf{S 1}$ for illustration of branches tested).

Results of the TLR7 branch-specific rate analyses (Table 2) suggest that the

165 Alouatta clade is under significantly stronger purifying selection than the rest of the primates in the phylogenetic tree $(p=0.057)$. TLR7 in the Old World primate lineage evolves under significantly less stringent purifying selection $\left(p=2.0 e^{-05}\right)$ than the New World primates. TLR7 branch-specific rate analysis using only A. palliata also found that Alouatta has significantly lower $d N / d S$

$170(p=0.046)$. This indicates that the first TLR7 result is not due to using three Alouatta species and one species for each other genus. The TLR8 branch-specific rate analyses show no evidence that TLR $8 d N / d S$ varies across the phylogenetic tree.

\subsection{Branch site test and functional analysis}

175 To identify specific codons subject to selection in the Alouatta lineage, we used a FEL test. Our null hypothesis was that the selection pressure for each codon is constant along the entire alignment. The alternative model allows the $d N / d S$ rate to vary codon by codon along the alignment. For this test, significance is reached at 0.10 (Kosakovsky Pond and Frost, 2005). Table 3 lists the codons 180 evolving under a significantly different $d N / d S$ rate in the Alouatta clade. FEL identified two sites under positive selection in TLR7 and six sites subject to positive selection in TLR8.

Within TLR7, codons with lower $d N / d S$ rates are due to a nonsynonymous variant in A. guariba clamitans (Table 3). The nonsynonymous change at

185 TLR7 codon 538 is within the pathogen binding region and causes an amino acid change to serine (SER) only in A. guariba clamitans. Every other primate in the alignment has the amino acid proline (PRO) at TLR7 codon 538. Proline is non-polar while serine is polar. The second positively selected site is in the TLR7 LRR. At TLR7 codon 721 a nonsynonymous change produces the amino acid threonine (THR) in A. guariba clamitans while every other primate in the alignment has the amino acid arginine (ARG) at this site. Threonine and arginine are both polar, but threonine is neutral and arginine is positively charged. FEL analysis identified six variants under selection in functionally relevant regions of TLR8 (Table 3). Five of these variants are found in all

195 three of the Alouatta species used in the study, and the last variant is unique to A. palliata. Five of the variants are within the LRR region of TLR8. The sixth variant is within the TIR domain.

\section{Discussion}

Previous studies evaluating evolutionary patterns of endosomal TLRs to identify 200 ${ }_{\text {}}$ pecies-specific differences have omitted the genus Alouatta in analyses Quach 
et al., 2013, Wlasiuk and Nachman, 2010). Our analyses of TLR7 and TLR8 DNA sequences in primates revealed strong purifying selection on TLR7 in the Alouatta clade and a number of unique sites under positive selection in both genes. This study provides the first insight into genetic factors that may contribute to Alouatta susceptibility to YFV.

We first tested TLR7 and TLR8 sequences for disparity in branch-specific selection in Alouatta compared to other New World and Old World primates with variable susceptibility to YFV. While it is known that TLR7 and TLR8 are under strong purifying selection due to their essential role in virus recogniWlasiuk and Nachman, 2010 Areal et our data showed that Alouatta TLR7 is subject to even stronger purifying selection than in other New World and Old World primate lineages. Since YFV originated in Africa and was introduced to the Americas by the slave trade recently, approximately 400 years ago (Bryant et al., 2007), Alouatta immune genes evolved under selection pressures from different ssRNA viruses. If the stringent purifying selection on TLR7 in the Alouatta lineage was necessary to maintain resistance to another virus, there would be little variation available to confer resistance to YFV, resulting in extreme susceptibility.

We also tested Alouatta TLR7 and TLR8 sequences for changes in codonspecific selection across the coding region. Despite strong purifying selection on TLR7, codon 528 in A. guariba clamitans is under positive selection. This site is in the pathogen binding region, meaning the amino acid directly interacts with ssRNA viruses, such as YFV, during infection (Wei et al., 2009). Amino acid differences within this region usually result from species-specific 225 selection pressure (Quach et al., 2013, Wlasiuk and Nachman, 2010). The second positively-selected codon in TLR7 (codon 721) is due to an amino acid difference in $A$. guariba clamitans within the extra-compartmental LRR region. While the codon is not in the direct binding region, the LRR also plays a role in pathogen sensing and stabilization during the recognition process (Wei et al.,

230 2009). Amino acid variations within TLR genes have been noted to change progression of a range of diseases in humans (Skevaki et al., 2015). Since both amino acid changes within TLR7 result in biochemical property changes at protein regions that interact with the pathogen, they are likely to affect binding pr stabilization of the pathogen, potentially affecting YFV progression (Skevaki) 235 et al. 2015). Both amino acid differences in TLR7 are unique to A. guariba clamitans. The TLR7 phylogenetic tree reflects the FEL finding of unique nonsynonymous changes in A. guariba clamitans, as the branch length is much longer than the other two Alouatta species due to a higher rate of substitution.

Future study of TLR7 in A. guariba clamitans individuals will be particularly important since the both positively selected variants identified in TLR7 are unique to the species. Additionally, the positively selected variants exist in spite of the resistance of TLR7 to substitutions in the Alouatta genus as a whole. A. guariba clamitans inhabits a region of Argentina with recent exposure to YFV due to deforestation (Di Bitetti et al., 1994; Holzmann et al., 2010). In

245 2007-2009, there was a YFV outbreak among the howler monkeys that resulted in mass deaths (Moreno et al., 2013, Holzmann et al., 2010; Bicca-marques and 
Freitas, 2010). Moving forward, it is imperative to collect samples from YFV affected Alouatta individuals to further explore whether the amino acid changes in A. guariba clamitans result in intragenus differences in YFV susceptibility previously unknown.

FEL identified six positively selected codons in TLR8. This is consistent with results from the branch-specific selection analysis, which found that TLR8 has been under less stringent purifying selection than TLR7 in Alouatta allowing more variation to arise in the gene. Five of the positively-selected codons in

255 TLR8 are within the LRR region and four substitutions result in a biochemical change. Unlike the positively selected sites in TLR7, five positively selected sites in TLR8 are due to an amino acid difference in all three Alouatta species. The LRR region substitutions that cause a biochemical change could result in altered YFV sensing and immune system signaling in the Alouatta genus, affecting susceptibility. These five amino acid differences in TLR 8 are found in all members of the Alouatta genus so it is likely that they arose earlier in Alouatta evolution before radiation and speciation across Central and South America. The sixth site, TLR7 codon 844, shows a nonsynonymous variant only in $A$. palliata. This site is within the TIR domain of the TLR. The TIR domain is

265 the inter-compartmental region of a TLR and is responsible for initiating the signaling cascade to activate the innate and adaptive immune systems upon infection (Mikami et al., 2012). Species-specific differences in the TIR region are much rarer than in other coding regions (Areal et al., 2011; Mikami et al., 2012). Thus, the finding of a positively selected variant in the more highly conserved region of TLR8 warrants further investigation.

Typically, a complex combination of environmental, pathogen and host genetic factors play a role in determining susceptibility to infection. Our results reveal a number of genetic factors that could impact YFV susceptibility in Alouatta. It is essential to collect additional samples from A. caraya and $A$. 275 guariba clamitans in YFV endemic ranges to clarify whether amino acid differences seen in this study are associated with YFV susceptibility. Moreover, further study may help identify genetic factors that influence susceptibility to YFV in humans and other primates.

\section{Acknowledgements}

${ }_{280}$ We would like to thank Andy Burrell, Christina Bergey, and Todd Disotell for allowing us to use their $A$. palliata reference genome. We would like to thank the Brian Dalley at the University of Utah Huntsman Cancer Institute High-Throughput Genomics Center and Mike Powers and Derek Warner at the University of Utah Sequencing Core for assistance with sequencing protocols. We would like to acknowledge Marcela Uhart, Hebe Ferreyra, and the curators at the Museo Argentina de Ciencias Naturales Bernardino Rivadavia for their contributions. Funding for this project was provided by the Center for Global Change and Sustainability at the University of Utah and the Wildlife Conservation Society. 


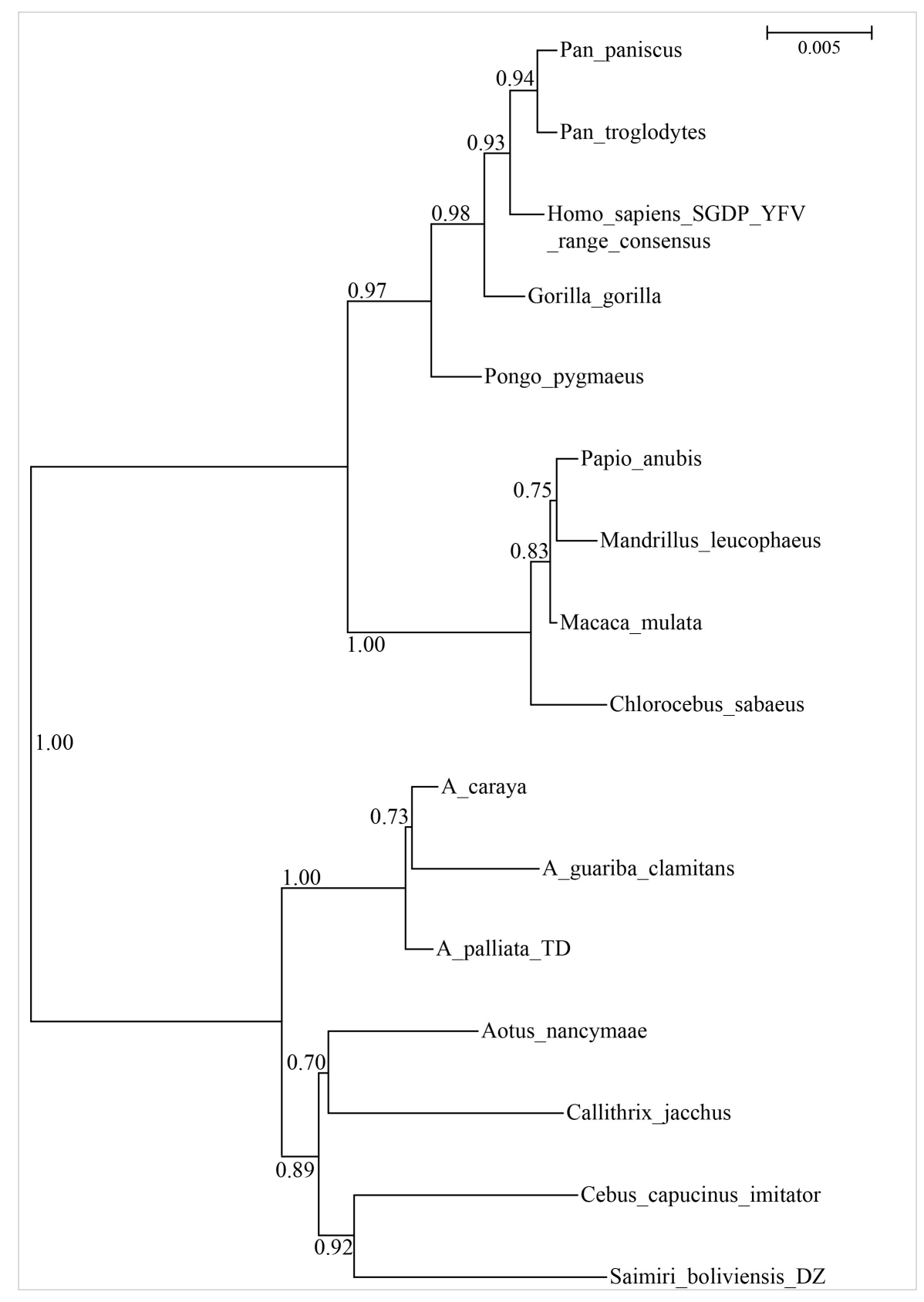

Figure 1: TLR7 maximum likelihood phylogenetic tree. TLR7 maximum likelihood phylogenetic tree generated from A. caraya and A. guariba clamitans TLR7 sequences (this study), New World and Old World primate TLR7 sequences. Each sequence totaled 3141 base pairs equaling 1039 codons. HKY model used, chosen by Decision Theoretic method. Log likelihood of the tree $=$ -6740.7 and aLRT support values on branches. 


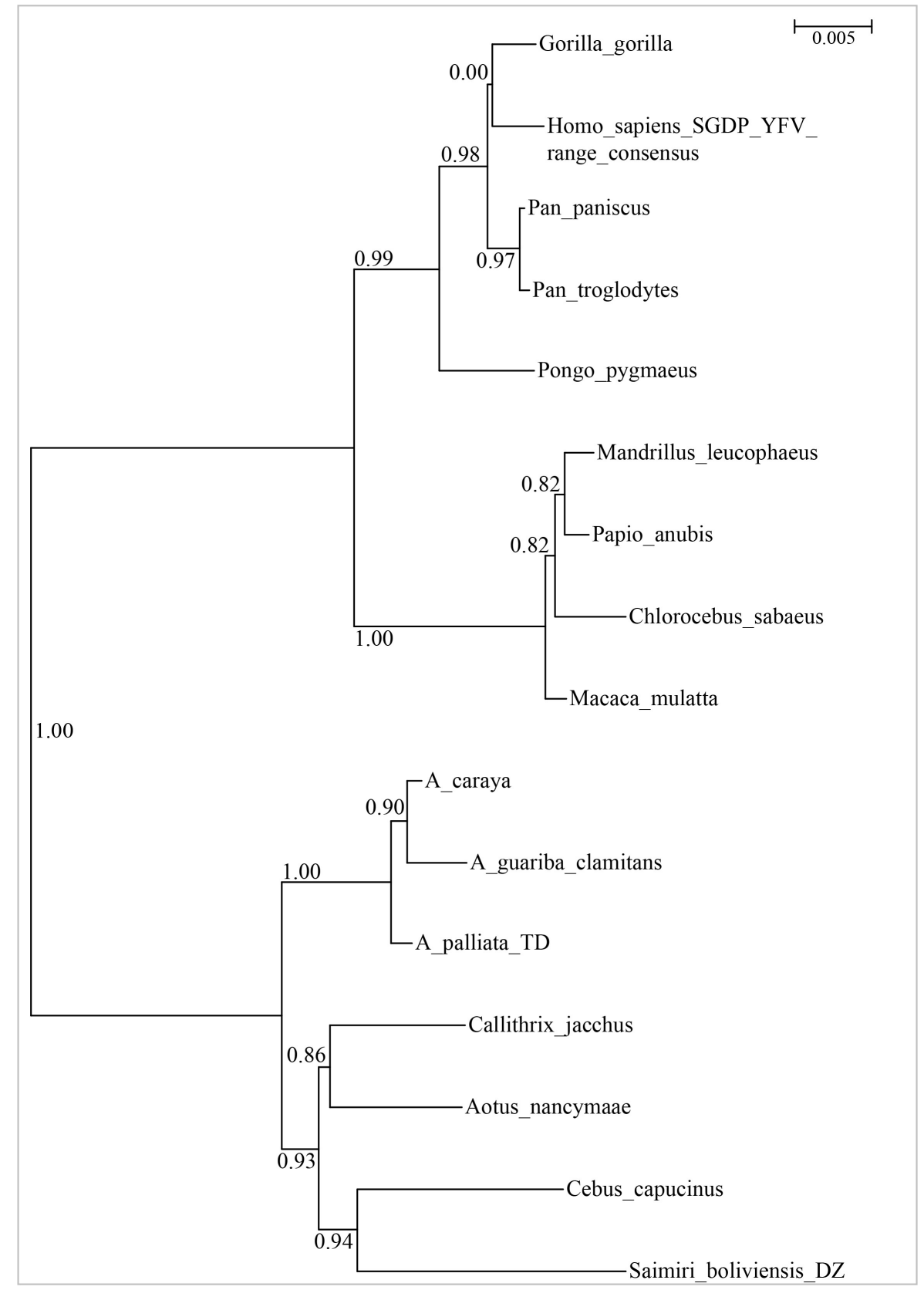

Figure 2: TLR8 maximum likelihood phylogenetic tree. TLR8 maximum likelihood phylogenetic tree generated from A. caraya and A. guariba clamitans TLR8 sequences (this study), New World and Old World primate TLR8 sequences. Each sequence totaled 3117 base pairs equaling 1047 codons. TN93 model used, chosen by Decision Theoretic method. Log likelihood of the tree $=$ -7145.9 and aLRT support values on branches. 
Table 1: Primate susceptibility to YFV, listed by increasing susceptibility (Bicca-marques and Freitas, 2010).

\begin{tabular}{lll} 
Family & Genus & Susceptibility \\
\hline Hominidae & Homo & Moderate \\
Cercopithecidae & All & Resistant \\
Cebidae & Cebus & \\
& Saimiri & \\
Callitrichidae & Saguinus & Moderate \\
& Callithrix & \\
Aotidae & Aotus & \\
Atelidae & Ateles & \\
& Alouatta & Extreme
\end{tabular}


bioRxiv preprint doi: https://doi.org/10.1101/708008; this version posted July 19, 2019. The copyright holder for this preprint (which was not certified by peer review) is the author/funder, who has granted bioRxiv a license to display the preprint in perpetuity. It is made available under aCC-BY-NC-ND 4.0 International license.

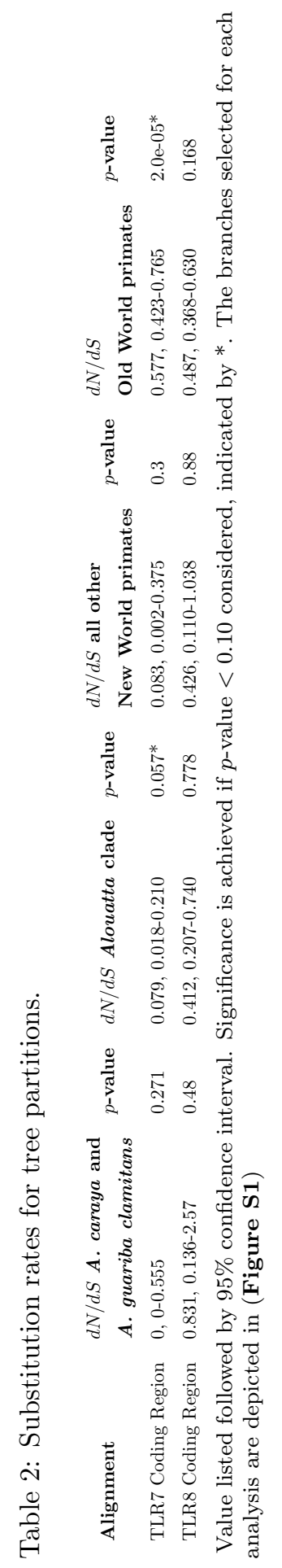


Table 3: Sites under positive selection in only in the howler monkey lineage identified by FEL analysis with functional implications.

\begin{tabular}{|c|c|c|c|c|c|c|c|}
\hline Gene & $\begin{array}{l}\text { Codon } \\
\text { Position }\end{array}$ & $p$-value & Alouatta Species & $\begin{array}{l}\text { AA in } \\
\text { Alouatta } \\
\text { species* }\end{array}$ & AA other primates & $\begin{array}{l}\text { Biochemical } \\
\text { property } \\
\text { difference } \\
(\mathrm{Y} / \mathrm{N})\end{array}$ & $\begin{array}{l}\text { Functional } \\
\text { Region }\end{array}$ \\
\hline TLR7 & 538 & 0.048 & AGC & SER & PRO & $\mathrm{Y}$ & Binding \\
\hline TLR7 & 721 & 0.097 & $\mathrm{AGC}$ & THR & ARG & $\mathrm{Y}$ & LRR \\
\hline TLR8 & 800 & 0.042 & AGC, AC, AP & ALA & VAL & $\mathrm{N}$ & LRR \\
\hline TLR8 & 248 & 0.055 & $\mathrm{AGC}, \mathrm{AC}, \mathrm{AP}$ & ILE & $\begin{array}{l}\text { ILA Siamiri, } \\
\text { THR all others }\end{array}$ & $\mathrm{Y}$ & LRR \\
\hline TLR8 & 284 & 0.063 & $\mathrm{AGC}, \mathrm{AC}, \mathrm{AP}$ & HIS & ASN & $\mathrm{Y}$ & LRR \\
\hline \multirow[t]{2}{*}{ TLR8 } & 844 & 0.065 & $\mathrm{AP}$ & VAL & ALA & $\mathrm{N}$ & TIR \\
\hline & & & & & THR & $\mathrm{Y}$ & \\
\hline TLR8 & 211 & 0.071 & $\mathrm{AGC}, \mathrm{AC}, \mathrm{AP}$ & TYR & CYS/SER & $\mathrm{Y}$ & LRR \\
\hline TLR8 & 744 & 0.097 & $\mathrm{AGC}, \mathrm{AC}, \mathrm{AP}$ & HIS & $\begin{array}{l}\text { HIS Callithrix, } \\
\text { TYR/SER }\end{array}$ & Y & LRR \\
\hline
\end{tabular}

Binding sites determined by (Wei et al. 2009). LRR regions determined by (Bell et al. 2003).

Abbreviations: $\mathrm{AGC}=$ A. guariba clamitans $; \mathrm{AC}=A$. caraya $; \mathrm{AP}=$ A. palliata $; \mathrm{AA}=$ amino acid

*As indicated in "Alouatta Species" column 


\section{References}

1000 Genomes Project Consortium, Auton, A., Brooks, L.D., Durbin, R.M., Garrison, E.P., Kang, H.M., Korbel, J.O., Marchini, J.L., McCarthy, S., McVean, G.A., Abecasis, G.R., 2015. A global reference for human genetic variation. Nature 526, 68-74. doi:10.1038/nature15393.

Anisimova, M., Gascuel, O., 2006. Approximate likelihood-ratio test for branches: A fast, accurate, and powerful alternative. Syst. Biol. 55, 539552. doi $10.1080 / 10635150600755453$.

Areal, H., Abrantes, J., Esteves, P.J., 2011. Signatures of positive selection in toll-like receptor (TLR) genes in mammals. BMC Evol. Biol. 11, 368. doi:10.1186/1471-2148-11-368.

Van der Auwera, G.A., Carneiro, M.O., Hartl, C., Poplin, R., Del Angel, G., Levy-Moonshine, A., Jordan, T., Shakir, K., Roazen, D., Thibault, J., Banks, E., Garimella, K.V., Altshuler, D., Gabriel, S., DePristo, M.A., 2013. From FastQ data to high confidence variant calls: the Genome Analysis Toolkit best practices pipeline. Curr. Protoc. Bioinformatics 43, 11.10.1-33. doi:10. 1002/0471250953.bi1110s43

Bankevich, A., Nurk, S., Antipov, D., Gurevich, A.A., Dvorkin, M., Kulikov, A.S., Lesin, V.M., Nikolenko, S.I., Pham, S., Prjibelski, A.D., Pyshkin, A.V., Sirotkin, A.V., Vyahhi, N., Tesler, G., Alekseyev, M.A., Pevzner, P.A., 2012. SPAdes: A new genome assembly algorithm and its applications to single-cell sequencing. J. Comput. Biol. 19, 455-477. doi 10.1089/cmb. 2012.0021.

Bell, J.K., Mullen, G.E.D., Leifer, C.A., Mazzoni, A., Davies, D.R., Segal, D.M., 2003. Leucine-rich repeats and pathogen recognition in toll-like receptors. Trends Immunol. 24, 528-533. doi:10.1016/S1471-4906(03)00242-4.

Bicca-marques, J.C., Freitas, D.S.D., 2010. The role of monkeys, mosquitoes, and humans in the occurrence of a yellow fever outbreak in a fragmented landscape in south Brazil: protecting howler monkeys is a matter of public health. Tropical Conservation Science 3, 78-89. doi $10.1177 / 194008291000300107$.

Boissinot, S., Tan, Y., Shyue, S.K., Schneider, H., Sampaio, I., Neiswanger, K., Hewett-Emmett, D., Li, W.H., 1998. Origins and antiquity of X-linked triallelic color vision systems in New World monkeys. Proc. Natl. Acad. Sci. U. S. A. 95, 13749-13754. doi:10.1073/pnas.95.23.13749.

Bolger, A.M., Lohse, M., Usadel, B., 2014. Trimmomatic: a flexible trim(1) mer for illumina sequence data. Bioinformatics 30, 2114-2120. doi 10.1093/ bioinformatics/btu170

Briggs, A.W., Stenzel, U., Johnson, P.L.F., Green, R.E., Kelso, J., Prüfer, K., Meyer, M., Krause, J., Ronan, M.T., Lachmann, M., Pääbo, S., 2007. Patterns of damage in genomic DNA sequences from a neandertal. Proc. Natl. Acad. Sci. U. S. A. 104, 14616-14621. doi:10.1073/pnas.0704665104. 
Bryant, J.E., Holmes, E.C., Barrett, A.D.T., 2007. Out of Africa: A molecular perspective on the introduction of yellow fever virus into the Americas. PLoS Pathog. 3, e75. doi 10.1371/journal.ppat.0030075.

Danecek, P., Auton, A., Abecasis, G., Albers, C.A., Banks, E., DePristo, M.A., Handsaker, R.E., Lunter, G., Marth, G.T., Sherry, S.T., McVean, G., Durbin, R., 1000 Genomes Project Analysis Group, 2011. The variant call format and VCFtools. Bioinformatics 27, 2156-2158. doi:10.1093/bioinformatics/ btr330.

Di Bitetti, M.S., Placci, G., Brown, A.D., Rode, D.I., 1994. Conservation and population status of the brown howling monkey (Alouatta fusca clamitans) in Argentina. Neotrop. Primates 2, 1-4.

Dudchenko, O., Batra, S.S., Omer, A.D., Nyquist, S.K., Hoeger, M., Durand, N.C., Shamim, M.S., Machol, I., Lander, E.S., Aiden, A.P., Aiden, E.L., 2017. De novo assembly of the Aedes aegypti genome using Hi-C yields chromosomelength scaffolds. Science 356, 92-95. doi $10.1126 /$ science.aal3327.

Enard, D., Cai, L., Gwennap, C., Petrov, D.A., 2016. Viruses are a dominant driver of protein adaptation in mammals. Elife 5. doi $10.7554 /$ eLife.12469

Frost, S.D.W., Liu, Y., Pond, S.L.K., Chappey, C., Wrin, T., Petropoulos, C.J., Little, S.J., Richman, D.D., 2005. Characterization of human immunodeficiency virus type 1 (HIV-1) envelope variation and neutralizing antibody responses during transmission of HIV-1 subtype B. J. Virol. 79, 6523-6527. doi:10.1128/JVI.79.10.6523-6527.2005.

Garrison, E., Marth, G., 2012. Haplotype-based variant detection from shortread sequencing arXiv:1207.3907.

Guindon, S., Dufayard, J.F., Lefort, V., Anisimova, M., Hordijk, W., Gascuel, O., 2010. New algorithms and methods to estimate maximum-likelihood phylogenies: Assessing the performance of PhyML 3.0. Syst. Biol. 59, 307-321. doi: $10.1093 /$ sysbio/syq010.

Hanley, K.A., Monath, T.P., Weaver, S.C., Rossi, S.L., Richman, R.L., Vasilakis, N., 2013. Fever versus fever: the role of host and vector susceptibility and interspecific competition in shaping the current and future distributions of the sylvatic cycles of dengue virus and yellow fever virus. Infect. Genet. Evol. 19, 292-311. doi $10.1016 /$ j.meegid.2013.03.008.

Harada, M.L.S., Schneider, H., Sampaio, M.P.C., Czelusniak, I., Goodman, M., 1995. DNA evidence on the phylogenetic systematics of New World mon365 keys: support for the sister-grouping of Cebus and Saimiri from two unlinked q nuclear genes. Mol. Phylogenet. Evol. 4, 331-349. doi 10.1006/mpev. 1995. 1029 . 
Holzmann, I., Agostini, I., Areta, J.I., Ferreyra, H., Beldomenico, P., di Bitetti, M.S., 2010. Impact of yellow fever outbreaks on two howler monkey species Alouatta guariba clamitans and (A. caraya) in Misiones, Argentina. Am. J. Primatol. 72, 475-480. doi:10.1002/ajp. 20796.

Jónsson, H., Ginolhac, A., Schubert, M., Johnson, P.L.F., Orlando, L., 2013. mapDamage2.0: fast approximate bayesian estimates of ancient DNA damage

parameters. Bioinformatics 29, 1682-1684. doi/10.1093/bioinformatics/ btt193.

Kawai, T., Akira, S., 2010. The role of pattern-recognition receptors in innate 口 immunity: update on toll-like receptors. Nat. Immunol. 11, 373-384. doi 10 . 1038/ni.1863.

Kosakovsky Pond, S.L., Frost, S.D.W., 2005. Not so different after all: a com380 parison of methods for detecting amino acid sites under selection. Mol. Biol. Evol. 22, 1208-1222. doi $10.1093 / \mathrm{molbev} / \mathrm{msi105}$.

Kosakovsky Pond, S.L., Frost, S.D.W., Muse, S.V., 2005. HyPhy: Hypoth口 esis testing using phylogenies. Bioinformatics 21, 676-679. doi 10.1093/ bioinformatics/bti079.

385 Kumar, H., Kawai, T., Akira, S., 2011. Pathogen recognition by the innate

口 immune system. Int. Rev. Immunol. 30, 16-34. doi 10.3109/08830185.2010. 529976.

Kumar, S., Stecher, G., Tamura, K., 2016. MEGA7: Molecular evolutionary genetics analysis version 7.0 for bigger datasets. Mol. Biol. Evol. 33, 18701874. doi $10.1093 / \mathrm{molbev} / \mathrm{msw054}$

Larsson, A., 2014. AliView: a fast and lightweight alignment viewer and

口 editor for large datasets. Bioinformatics 30, 3276-3278. doi 10.1093/ bioinformatics/btu531.

Li, H., 2011. A statistical framework for SNP calling, mutation discovery, association mapping and population genetical parameter estimation from se-

घ quencing data. Bioinformatics 27, 2987-2993. doi/10.1093/bioinformatics/ btr509.

Li, H., 2013. Aligning sequence reads, clone sequences and assembly contigs with BWA-MEM arXiv:1303.3997.

400 Li, H., Durbin, R., 2009. Fast and accurate short read alignment with

ㅁ Burrows-Wheeler transform. Bioinformatics 25, 1754-1760. doi 10.1093/ bioinformatics/btp324.

Li, H., Handsaker, B., Wysoker, A., Fennell, T., Ruan, J., Homer, N., Marth, G., Abecasis, G., Durbin, R., 1000 Genome Project Data Processing Subgroup, 2009. The sequence Alignment/Map format and SAMtools. Bioinformatics 25, 2078-2079. doi:10.1093/bioinformatics/btp352. 
Lin, Y.T., Verma, A., Hodgkinson, C.P., 2012. Toll-like receptors and human disease: lessons from single nucleotide polymorphisms. Curr. Genomics 13, 633-645. doi:10.2174/138920212803759712.

Mallick, S., Li, H., Lipson, M., Mathieson, I., Gymrek, M., Racimo, F., Zhao, M., Chennagiri, N., Nordenfelt, S., Tandon, A., Skoglund, P., Lazaridis, I., Sankararaman, S., Fu, Q., Rohland, N., Renaud, G., Erlich, Y., Willems, T., Gallo, C., Spence, J.P., Song, Y.S., Poletti, G., Balloux, F., van Driem, G., de Knijff, P., Romero, I.G., Jha, A.R., Behar, D.M., Bravi, C.M., Capelli, C., Hervig, T., Moreno-Estrada, A., Posukh, O.L., Balanovska, E., Balanovsky, O., Karachanak-Yankova, S., Sahakyan, H., Toncheva, D., Yepiskoposyan, L., Tyler-Smith, C., Xue, Y., Abdullah, M.S., Ruiz-Linares, A., Beall, C.M., Di Rienzo, A., Jeong, C., Starikovskaya, E.B., Metspalu, E., Parik, J., Villems, R., Henn, B.M., Hodoglugil, U., Mahley, R., Sajantila, A., Stamatoyannopoulos, G., Wee, J.T.S., Khusainova, R., Khusnutdinova, E., Litvinov, S., Ayodo, G., Comas, D., Hammer, M.F., Kivisild, T., Klitz, W., Winkler, C.A., Labuda, D., Bamshad, M., Jorde, L.B., Tishkoff, S.A., Watkins, W.S., Metspalu, M., Dryomov, S., Sukernik, R., Singh, L., Thangaraj, K., Pääbo, S., Kelso, J., Patterson, N., Reich, D., 2016. The Simons Genome Diversity Project: 300 genomes from 142 diverse populations. Nature 538, 201-206. doi $10.1038 /$ nature18964

Mikami, T., Miyashita, H., Takatsuka, S., Kuroki, Y., Matsushima, N., 2012. Molecular evolution of vertebrate toll-like receptors: evolutionary rate difference between their leucine-rich repeats and their TIR domains. Gene 503, 235-243. doi:10.1016/j.gene.2012.04.007.

Moreno, E.S., Spinola, R., Tengan, C.H., Brasil, R.A., Siciliano, M.M., Coimbra, T.L.M., Silveira, V.R., Rocco, I.M., Bisordi, I., de Souza, R.P., Petrella, S., Pereira, L.E., Maeda, A.Y., da Silva, F.G., Suzuki, A., 2013. Epizootias de febre amarela em primatas não humanos no estado de São

435 Paulo, Brasil, 2008-2009. Rev. Inst. Med. Trop. Sao Paulo 55, 45-50. doi:10.1590/S0036-46652013000100008.

Muse, S.V., Gaut, B.S., 1994. A likelihood approach for comparing synonymous and nonsynonymous nucleotide substitution rates, with applica-

a tion to the chloroplast genome. Mol. Biol. Evol. 1, 715-724. doi 10.1093/ oxfordjournals.molbev.a040152.

Pozzi, L., Hodgson, J.A., Burrell, A.S., Sterner, K.N., Raaum, R.L., Disotell, T.R., 2014. Primate phylogenetic relationships and divergence dates inferred from complete mitochondrial genomes. Mol. Phylogenet. Evol. 75, 165-183. doi $10.1016 / j \cdot$ ympev.2014.02.023.

${ }_{445}$ Quach, H., Wilson, D., Laval, G., Patin, E., Manry, J., Guibert, J., Barreiro, L.B., Nerrienet, E., Verschoor, E., Gessain, A., Przeworski, M., QuintanaMurci, L., 2013. Different selective pressures shape the evolution of toll-like 
receptors in human and African great ape populations. Hum. Mol. Genet. 22, 4829-4840. doi $10.1093 / \mathrm{hmg} / \mathrm{ddt} 335$

450

n. bi1112s47.

Rassa, J.C., Ross, S.R., 2003. Viruses and toll-like receptors. Microbes Infect. 5, 961-968. doi $10.1016 / \mathrm{S} 1286-4579(03) 00193-\mathrm{X}$.

455 Schad, J., Voigt, C.C., 2016. Adaptive evolution of virus-sensing toll-

n like receptor 8 in bats. Immunogenetics 68, 783-795. doi 10.1007/ s00251-016-0940-z.

Sironi, M., Cagliani, R., Forni, D., Clerici, M., 2015. Evolutionary insights into host-pathogen interactions from mammalian sequence data. Nat. Rev. Genet. 16, 224-236. doi $10.1038 / \mathrm{nrg} 3905$.

Skevaki, C., Pararas, M., Kostelidou, K., Tsakris, A., Routsias, J.G., 2015. Single nucleotide polymorphisms of toll-like receptors and susceptibility to

n infectious diseases. Clin. Exp. Immunol. 180, 165-177. doi:10.1111/cei. 12578 .

465 Steiper, M.E., Ruvolo, M., 2003. New World monkey phylogeny based on X-

linked G6PD DNA sequences. Mol. Phylogenet. Evol. 27, 121-130. doi 10 . 1016/S1055-7903(02)00375-5.

Sullivan, J., Joyce, P., 2005. Model selection in phylogenetics. Annu. Rev. Ecol. Evol. Syst. 36, 445-466. doi:10.1146/annurev.ecolsys.36.102003.152633.

470 Swofford, D.L., 2003. PAUP*. phylogenetic analysis using parsimony (*and other methods).

Wei, T., Gong, J., Jamitzky, F., Heckl, W.M., Stark, R.W., Rössle, S.C., 2009. Homology modeling of human toll-like receptors TLR7, 8 , and 9 ligandbinding domains. Protein Sci. 18, 1684-1691. doi:10.1002/pro.186.

475 Wlasiuk, G., Nachman, M.W., 2010. Adaptation and constraint at toll-like 口 receptors in primates. Mol. Biol. Evol. 27, 2172-2186. doi 10.1093/molbev/ msq104. 
bioRxiv preprint doi: https://doi.org/10.1101/708008; this version posted July 19, 2019. The copyright holder for this preprint (which was not certified by peer review) is the author/funder, who has granted bioRxiv a license to display the preprint in perpetuity. It is made available under aCC-BY-NC-ND 4.0 International license.

\section{Supplement}


bioRxiv preprint doi: https://doi.org/10.1101/708008: this version posted July 19,2019 . The copyright holder for this preprint (which was not certified by peer review) is the author/funder, who has granted bioRxiv a license to display the preprint in perpetuity. It is made available under aCC-BY-NC-ND 4.0 International license.

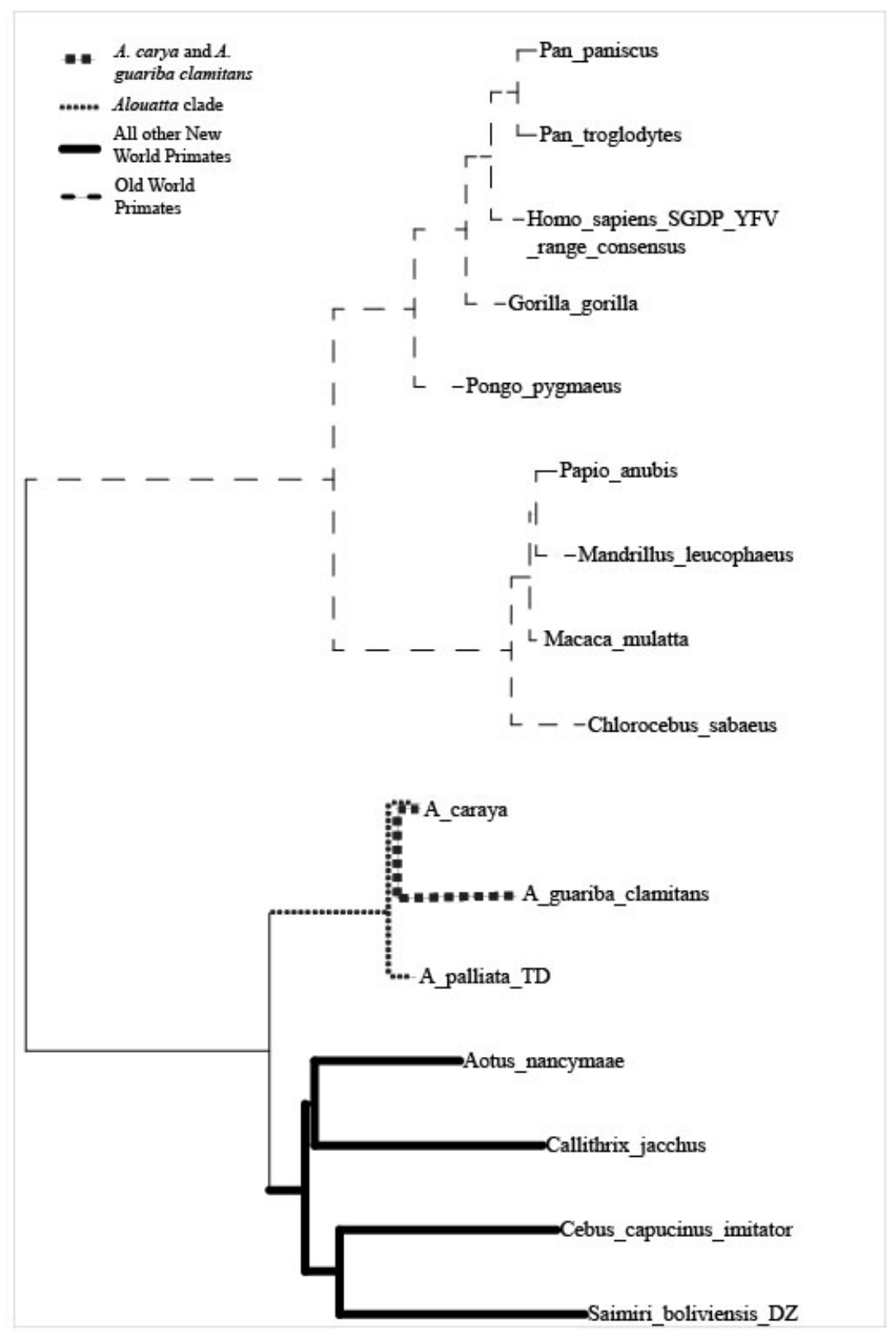

Figure S1: Illustration of branch bipartitions selected for SelectionLRT and FEL analyses. 


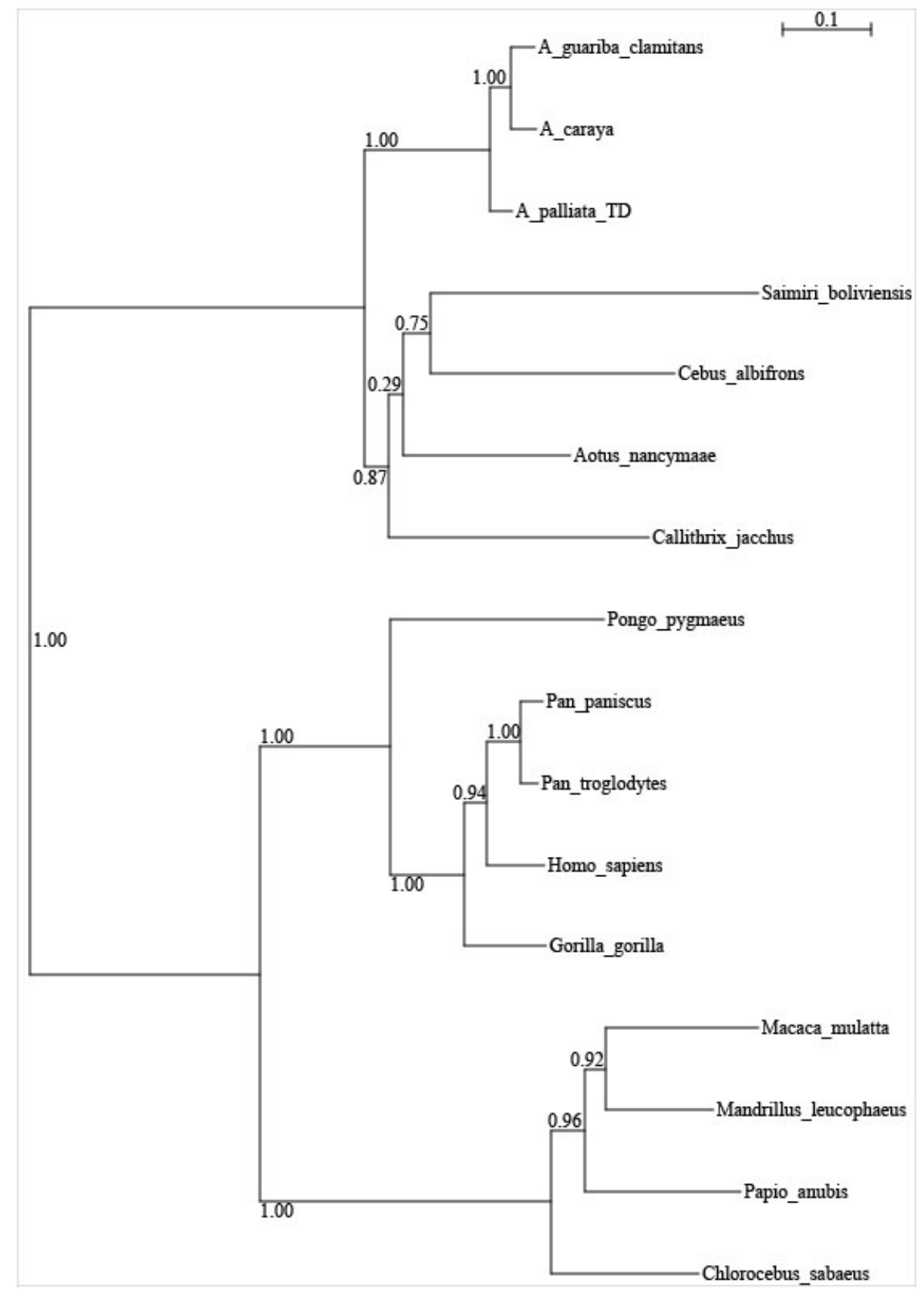

Figure S2: ND1-ND2-CO1 maximum likelihood phylogenetic tree generated from A. caraya and A. guariba clamitans TLR8 sequences (this study), New World and Old World primate mtDNA sequences Table S3. HKY model used, chosen by Decision Theoretic method. Each sequence totaled 3541 base pairs. Log likelihood of the tree $=-24404.7$ and aLRT support values on branches. 
Table S1: Sample metadata

\begin{tabular}{|c|c|c|c|c|c|c|c|}
\hline Sample & Species & Collection location & Collection date & Sample type & Obtained from & Source ID & Sex \\
\hline AC_tissue & A. caraya & Pindapoy Arroyo, San Jose & 2009 & liver tissue & Hernan Argibay & $\mathrm{AC} 10$ & M \\
\hline AC_Museum & A. caraya & Corrientes, Argentina & 1967 & taxidermied skin & $\begin{array}{l}\text { Museo Argentina de Ciencias } \\
\text { Naturales Bernardino Rivadavia }\end{array}$ & 13901 & $\mathrm{~F}$ \\
\hline AGC_Museum & A. guariba clamitans & Misiones, Argentina & 1952 & taxidermied skin & $\begin{array}{l}\text { Museo Argentina de Ciencias } \\
\text { Naturales Bernardino Rivadavia }\end{array}$ & 52.41 & $\mathrm{M}$ \\
\hline
\end{tabular}

Table S2: GenBank Accession Numbers

$\begin{array}{lll}\text { Sample } & \text { Gene } & \text { GenBank accession } \\ \text { AC_tissue } & \text { TLR7 } & \text { MN046896 } \\ \text { AC_tissue } & \text { TLR8 } & \text { MN046897 } \\ \text { AC_tissue } & \text { ND2 } & \text { MN046902 } \\ \text { AC_tissue } & \text { ND1 } & \text { MN046903 } \\ \text { AC_tissue } & \text { CO1 } & \text { MN046904 } \\ \text { AC_museum } & \text { TLR7 } & \text { MN046898 } \\ \text { AC_museum } & \text { TLR8 } & \text { MN046899 } \\ \text { AC_museum } & \text { ND2 } & \text { MN046905 } \\ \text { AC_museum } & \text { ND1 } & \text { MN046906 } \\ \text { AC_museum } & \text { CO1 } & \text { MN046907 } \\ \text { AGC_museum } & \text { TLR7 } & \text { MN072369, MN072370 } \\ \text { AGC_museum } & \text { TLR8 } & \text { MN046901 } \\ \text { AGC_museum } & \text { ND2 } & \text { MN046908 } \\ \text { AGC_museum } & \text { ND1 } & \text { MN046909 } \\ \text { AGC_museum } & \text { CO1 A } & \text { MN045881 } \\ \text { AGC_museum } & \text { CO1 B } & \text { MN045882 }\end{array}$


Table S3: GenBank Accession numbers for TLR7 and TLR8 sequences used in phylogenetic analysis.

\begin{tabular}{|c|c|c|c|}
\hline Species & TLR7 GenBank Accession & TLR8 GenBank Accession & mtDNA \\
\hline Cebus capucinus & XM 017508809.1 & XM 017508811.1 & not available \\
\hline Cebus albifrons & & & NC_002763.1 \\
\hline Aotus nancymaae & XM 012435148.1 & XM 012435138.2 & NC_018116.1 \\
\hline Chlorocebus sabaeus & XM 007991035.1 & XM 007991038.1 & EF597503 \\
\hline Mandrillus leucophaeus & XM 011994858.1 & XM 011994857.1 & NC_028442 \\
\hline Pongo pygmaeus & AB445663.1 & AB445670.1 & NC_001646.1 \\
\hline Papio anubis & XM 021932909.1 & XM 009197197.3 & MG787545.1 \\
\hline Macaca mulatta & EU204943.1 & EU204944.1 & NC_005943 \\
\hline Gorilla gorilla & KF321036.1 & KF321278.1 & NC_001645.1 \\
\hline Pan paniscus & XM 003805682.2 & XM 008973908.2 & NC_001644.1 \\
\hline Saimiri boliviensis & DNAzoo.org $^{\dagger}$ & DNAzoo.org $^{\dagger}$ & КС959987.1 \\
\hline Pan troglodytes & KF321089.1 & KF321320.1 & D38113.1 \\
\hline \multirow[t]{2}{*}{ Callithrix jacchus } & XM 002762618.4 & XM 008988948.2 & KM588314 \\
\hline & \multicolumn{3}{|l|}{ Variants from SGDP dataset } \\
\hline \multirow[t]{2}{*}{ Homo sapiens } & Mallick et al., 2016 applied to & 5) applied to hg19 reference & NC_012920.1 \\
\hline & \multicolumn{3}{|c|}{1000 Genomes Project Consortium et al., 2015) } \\
\hline
\end{tabular}

${ }^{\dagger}$ This assembly was generated by the DNA Zoo team in collaboration with Jessica Alfoldi, Broad Institute, using an early version of DISCOVAR de novo contigger.

Table S4: Tree parameters: Phylogenetic tree parameters for each alignment as determined by Decision Theoretic method in PAUP (Swofford, 2003).

$\begin{array}{lllll}\text { Alignment } & \text { Model } & \text { Ts/Tv ratio } & \begin{array}{l}\text { Invariable sites/ } \\ \text { pinv }\end{array} & \begin{array}{l}\text { Across-site rate } \\ \text { variation/ } \\ \text { Gamma rate }\end{array} \\ \text { TLR7 Coding Region } & \text { HKY } & 4.54 & 0.747 & 0.171 \\ \text { TLR8 Coding Region } & \text { TN } & \text { Optimized } & 0.685 & 0.164 \\ \text { mtDNA: CO1-ND1-ND2 } & \text { HKY } & 27.33 & 0.391 & 0.32\end{array}$

Table S5: Read depth for at each gene and exon of $A$. caraya sample shotgun sequenced.

\begin{tabular}{lll} 
Gene & Exon & Average Read Depth \\
\hline TLR7 & 1 & 9.13 \\
& 2 & 12.45 \\
& 3 & 10.6 \\
\hline TLR8 & 1 & 7.53 \\
& 2 & 10.68
\end{tabular}


Table S6: Read depth for at each gene and exon of the A. caraya and $A$. guariba clamitans museum samples.

\begin{tabular}{|c|c|c|c|c|}
\hline Sample & Gene & Exon & Average Read Depth & $\begin{array}{l}\text { Average Read Depth } \\
\text { after removing duplicates }\end{array}$ \\
\hline \multirow[t]{5}{*}{ AC_Museum } & TLR7 & 1 & 516.96 & 88.78 \\
\hline & & 2 & 5050.54 & 289.12 \\
\hline & & 3 & 1257.26 & 214.78 \\
\hline & TLR8 & 1 & 349.05 & 41.47 \\
\hline & & 2 & 1037.97 & 182.96 \\
\hline \multirow[t]{5}{*}{ AGC_Museum } & TLR7 & 1 & 21.94 & 6.42 \\
\hline & & 2 & 80.83 & 9.07 \\
\hline & & 3 & 43.98 & 8.33 \\
\hline & TLR8 & 1 & 10.51 & 3.94 \\
\hline & & 2 & 60.63 & 9.74 \\
\hline
\end{tabular}

Table S7: MapDamage2.0 results for museum samples.

\begin{tabular}{ll} 
Sample & Output \\
\hline AC_Museum & 0.00374 \\
AG_Museum & 0.00485
\end{tabular}

Values indicate the difference in $\mathrm{C}>\mathrm{T}$ substitutions between the sample and the reference at the first 5' position. Values less than 0.01 indicate that level of DNA damage is low (Jónsson et al. 2013). 


\section{Methods}

We processed shotgun sequencing results and created a reference sequence using the following pipeline and commands:

1. Remove Illumina adapters from forward and reverse sequencing data (Bolger et al., 2014).

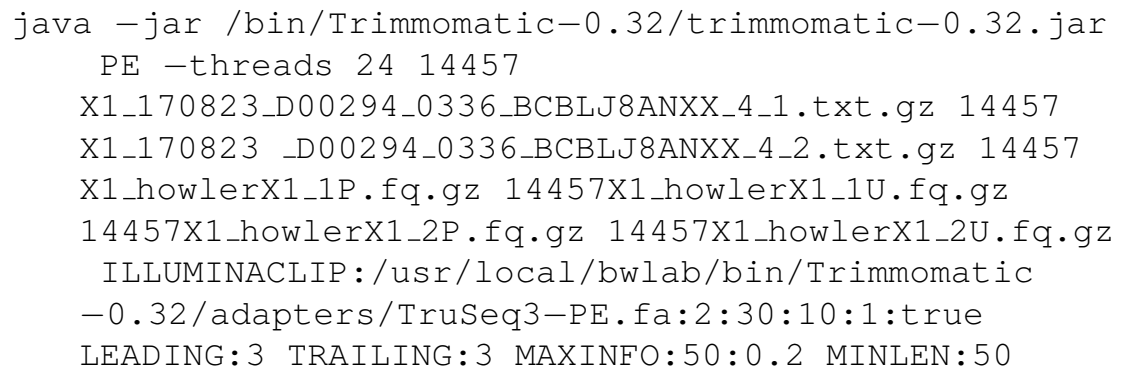

2. Align forward and reverse howler monkey reads to a masked version of the human reference genome (hg19) 1000 Genomes Project Consortium et al. 2015) to reduce repetitive sequences (Li and Durbin, 2009). Output interlaced paired end read alignment.

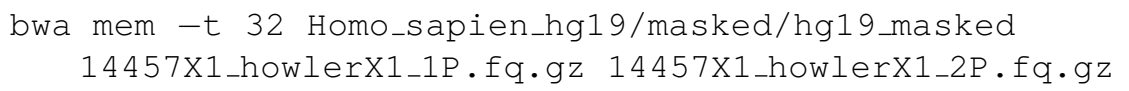

3. Sort the aligned BAM files (Li, 2011).

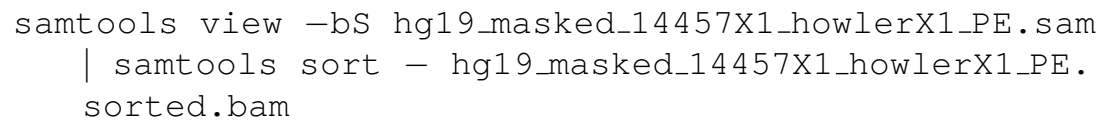

4. BAM files filtered to target aligned reads to TLR7 and TLR 8 and only include reads of quality MAPQ $>60(\mathrm{Li}, 2011)$.

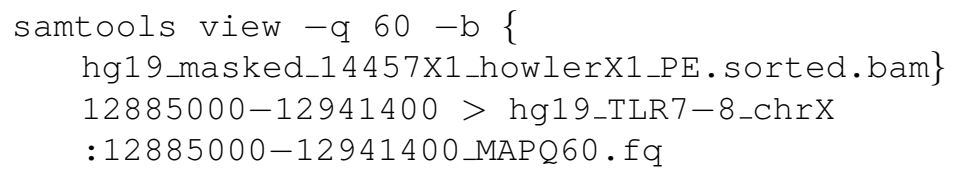

5. Create a consensus sequence of TLR7 and TLR8 using the fastq files (Bankevich et al., 2012).

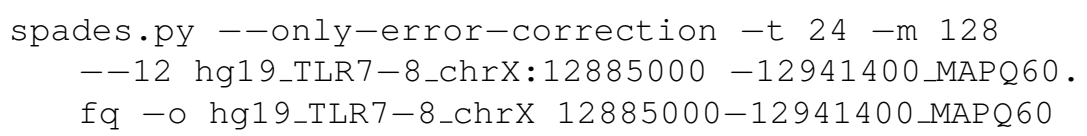


TLR7 and TLR 8 are extremely conserved, especially within primates, therefore the howler monkey TLR7 and TLR8 genes were expected to align to the human reference genome. The same pipeline was used to align Alouatta caraya sequencing data to an A. palliata reference sequence provided by the A. Burrell at New York University. The final A. caraya TLR7 and TLR8 reference genomes created were identical.

We processed targeted sequencing results using the following pipeline:

1. Unalign BAM files for AC_museum and AGC_museum using GATK software (Van der Auwera et al., 2013).

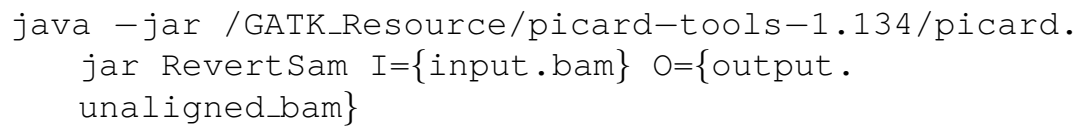

2. Convert unaligned BAM files to Fastq format (Quinlan, 2014).

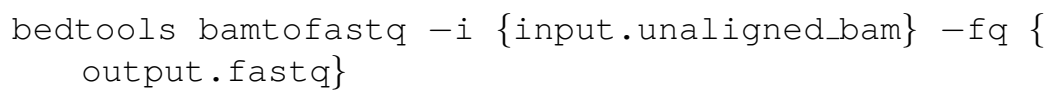

3. Assess fastq quality. Software available at http://www.bioinformatics.babraham.ac.uk/projects/fastqc/. fastqc -o fastqc_results sample.fastq

4. Trim fastq reads based on fastq quality results. Software available at https://sourceforge.net/projects/bbmap/.

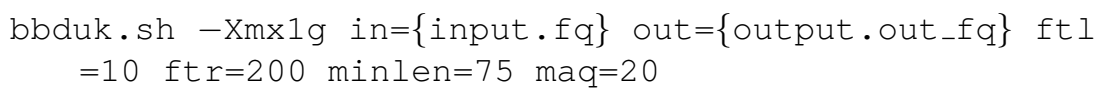

7. Realign trimmed Fastq files for each sample to AC_tissue reference (Li and Durbin, 2009).

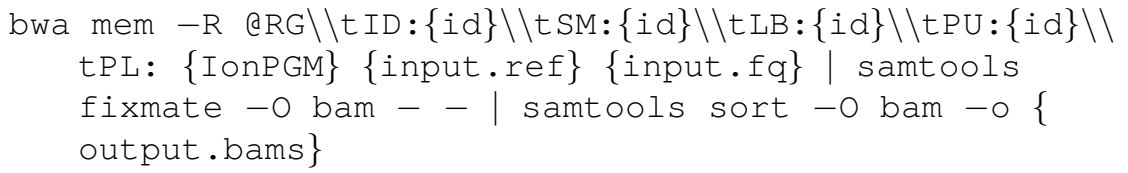

6. Index each BAM file (Li, 2011).

samtools index $\{$ input

7. Mark duplicate reads using GATK software (Danecek et al., 2011).

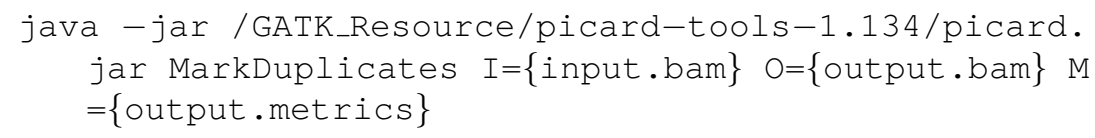


8. Create a consensus VCF based on the BAM files (with duplicate reads marked) of each species (Garrison and Marth, 2012).

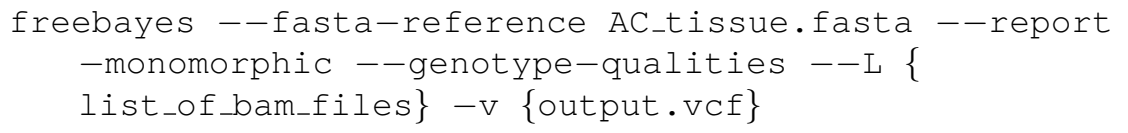

9. Remove indels from VCF file and filter by genotype quality (Danecek et al. 2011). Indels were removed since they appeared to be related to sequencing error caused by long strings of nucleotide Adenine.

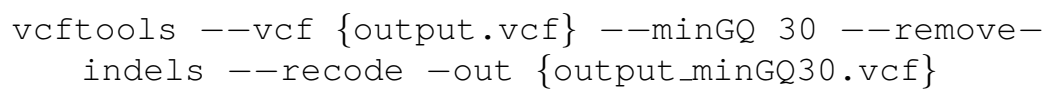

10. Create a consensus sequence for the A. caraya and A. guariba clamitans taxidermied skin samples (Li et al., 2009). 